\title{
How to Make Business with Computer Vision Technology
}

\author{
Giovanni B. Garibotto \\ Elsag spa, Genova, Italy \\ giovanni.garibotto@elsag.it
}

\begin{abstract}
Business development in highly competitive new markets is strongly dependent on the level of technology innovation. Actually, the real success of a new product is affected by many other factors in the development chain, including customer requirements understanding and system implementation. The paper is aimed to refer some direct experience of New product development based on Computer Vision Technology. A practical example is referred to describe a recent success story in the field of Security application, a mobile Automatic Number Plate Recognition system that is currently used by many security forces in different countries in Europe and the USA.
\end{abstract}

\section{Business Development by Technology Innovation}

There is a widespread agreement that technology innovation is a key to economic growth. Actually social and political forces determine the context within which R\&D technology investments occur and strongly influence perceived rewards, risks and tradeoffs. Industry-market trends and factors determine opportunities due to market maturity, number of competitors, etc. Rouse [1] concludes that company's abilities to recognize changing relationships with evolving markets are key factors to continued success. The time until returns is usually quite long. Academic research often requires 20 years until economic gain [2]. Actually much shorter time horizons are usually considered as useful, especially in the industry where most application programs are often limited to yearly budgets.

Moreover, R\&D technology efforts tend to achieve the greatest benefits in unanticipated domains and markets [1]. A key issue is the identification of the role of R\&D technology in the overall organization.

Many managers in the past saw the R\&D function as a knowledge incubator, where a wide range of ideas are pursued and various invention developed. A much more agreed answer today is considering R\&D function to provide enabling technologies to support market penetration. Moreover, it should give key competitive advantages by creating leading-edge products and processes. Thus, R\&D objectives are more directly linked to business objectives than in the past.

Another important question is related to the capability to measure success of R\&Dtechnology innovation. Traditional metrics based on the number of publications and patents are no more sufficient. Much more emphasis is given on measurable value added solutions of technical and operational problems (product and process innovation). 


\subsection{Technology Transfer Actions}

At the international level many institutions are already promoting technology transfer from basic research to product development. One example is given by the IST innovation prize that has been organized by EEC [3] to promote innovation and technology transfer activities, from the University to the industry and to the market. Traditional university structures have not been designed for technology transfer purposes but for research and education only. As a result, there are often organization constraints that limit universities' abilities to carry out technology transfer efficiently with industrial companies or to commercialize the results of their research. A few relevant changes are required in the academic context to fulfill pre-requisites for efficient and successful collaborations with industry. Main challenges are to move from a given budget to more competitive markets, from scientific curiosity to concrete market needs, from continuity to improved flexibility, from bureaucracy to management efficiency, from the ivory tower of science to international competition.

The most common model of technology transfer process is the linear model that has been used as a grounding theory for all science parks and incubators. According to the linear model the process starts with basic scientific research and passes through applied and more developmental research activities, to achieve the identification of new products and new process ideas, the evolution and testing of prototypes, to get commercial production and finally to achieve and consolidate an effective market position. The linear model is quite appropriate to highlight the relationship between long-term scientific research and its commercialization and continues to dominate much science and technology policy-making [4]. Anyway, a few main critical issues have been found to linear models. Actually, research results and ideas are used at all stages of the innovation process and not only in the early phase and the relationship between basic research and commercialization is too complex to be described as a linear function and requires a series of feedback loops and changing actions to succeed. Finally, the linear models tend to underestimate the contributions of people involved in the innovation process, including users whose ideas are often another relevant starting point for innovation.

\subsection{Evaluating a Technology Transfer Project}

It is important to evaluate technology transfer programs and projects on an ongoing basis to determine their success and to incorporate lessons learned in future activities. Recent studies of just over 400 examples of the application of technology from the National Aeronautics and Space Administration (NASA) demonstrated that the technology users benefited from sales or savings of $\$ 33.5$ billion because of successful technology transfer. In a similar study for the Agricultural Research Service (ARS), sales or savings totaling $\$ 14.3$ billion were identified in 87 cases where users applied ARS technology or science. Technology transfer is rarely accomplished in a short period of time. The process may not be complete for five or ten years. Therefore, it is not always possible to wait for the end results; it is sometimes necessary to seek interim indicators that will demonstrate progress toward the particular project goal. It is important to identify intermediate products whenever possible. For example, a project that uses remediation technology might include plans 
to use a variety of workshops and demonstrations as interim steps. Some experts say a transfer is successful only when it becomes a profitable product or process, while others claim a transfer is successful when the technology is at least reviewed for possible use by another person or organization.

\section{Computer Vision Market}

Different areas of markets are currently addressed by Image Processing and Computer Vision technology. A very simple and basic main classification of the different market sectors can be arranged as follows:

- Image quality inspection for industrial applications (industrial automation, pharmaceutical and manufacturing, etc.) represents a mature market field with many specialized Exhibition and Congress.

- OCR (Optical Character Recognition) in postal automation and document processing represents still one of the most important business success of Image Processing and Recognition technologies.

- Biomedical Image Processing for Diagnostic systems and surgical systems is an essential component of all new advanced devices in the medical field.

- Robotics, Automation in different areas (Space, applications, service robotics and Logistic automation)

- Image Processing in Telecommunications and Multimedia represents another relevant component of technology innovation

- Security and Surveillance Applications is the emerging area with particular interest in video-surveillance, face-recognition and biometric control, human motion analysis and behavior control.

\section{A Success Story: Auto-Detector}

Auto-Detector [5] is a new mobile Automatic Number Plate Recognition system, installed on board of any kind of patrol vehicle (car, motor-vehicle), to automatically detect and read the license plates of the vehicles falling in its field of view. The innovation content of Auto-Detector is twofold: it is a new service solution for security and surveillance monitoring; moreover it represents a significant innovation of integrated Computer Vision solutions by continuous high-rate number plate recognition from a moving sensor in all possible environmental conditions.

Auto-Detector is an independent intelligent sensor that is continuously inspecting what happens around the patrol vehicle and is able to detect automatically the presence of a license plate irrespective of its orientation in the field of view. As such the proposed system represents a revolutionary contribution to patrol crew, working in background and without affecting normal patrol duties.

The continuous recognition of plates in the scene is a performance far exceeding any practical possibility by the human eye and the on-line response feature of AutoDetector (by on-board real-time checking the recognized plate against a search list size of more than millions of plates) provide a great economical value for all security and surveillance applications. All recognized license plates are immediately compared 
on board, against one or more lists of selected number-plates according to different levels of investigation priorities. They may be either alarm hot-list (including plates under investigation, as stolen cars or searched vehicles) as well white list of authorized vehicles (access control to downtown restricted areas).

\subsection{The Industrial Context}

Elsag spa is a benchmark player in the Italian Information \& Communication Technology market, providing services and solutions in pursuit of its mission to "Satisfy the requirements of medium and large companies by adopting innovative technologies to design, implement and manage ICT services and solutions". ELSAG Company has been established in 1905. It is a medium-large size company with approximately 2950 employees (2003). Elsag belongs to Finmeccanica spa with leadership in Information Technology, in the fields of Automation, Information and Physical Security and Defense. In the last few years there has been a significant development of new surveillance applications in the traffic control market, with an increasing connection to institutional security customers (Ministry of the Defense and Ministry of the Interior and police departments). Elsag is qualified as a high level system integration of complex and high performance systems, with Information Technology components. Because of this system integration approach, the development of new products is always considered as an added value component of the Elsag's offer in the complete integrated system.

\subsection{Innovation Content of Computer Vision and Image Processing}

The main contribution of technology innovation in the Auto-Detector project is provided in the Computer Vision process and Optical Character Recognition to achieve a detection performance better than $90 \%$ of all license plates in the field of view and a correct recognition rate greater than 99\% among all detected license plates. Moreover the system has been equipped also with suitable and effective learning tools, to achieve successful performance for all international number plates in all countries. One of the key features of the Auto-Detector is the high-performance Elsag $\mathrm{O}^{2} \mathrm{CR}$ technology that has been strongly improved to read License Plates continuously from the video stream at the highest rate (more than 25 frame per second fps, for progressive cameras) without any need of external triggering devices. This technology is based on Elsag's long experience in Intelligent Character Recognition applied to document and form processing as well as for postal address understanding.

Adaptive exposure control is an essential feature of the acquisition system. Computer Vision and Image Processing technologies are used to achieve the most effective image normalization and optimize character recognition (in a mobile application License Plates may appear with a perspective 3D deformation greater than $30^{\circ}$ ). The recognition process consists in three main blocks: Plate detection, Recognition Measure and Contextual Analysis (exploiting both spatial and syntactic information to select the best hypothesis). Temporal post-processing performs data fusion and tracking among different images of the video sequence.

Another important innovation contribution is the miniaturization of the imaging sensor case, to be installed on-board within a very small space (lighting bar, roof of 
the car, interior, etc.). To achieve such goals the proposed solution is an effective optimization of existing technology components in the area of digital cameras (LVDS and Camera-Link standard) and infrared illumination sources (using a very innovative LED-on-chip technology that was originally developed and used for automation inspection and Machine Vision). Finally, the selected Auto-Detector on-board processing unit is an effective network processor using a local LAN connection between dedicated processors devoted to each camera and data storage and search, with low-power consumption (less than $15 \mathrm{~W}$ ), and automotive constraints.

\subsection{Main System Features}

The use of a mobile License Plate Reader (LPR) system provides some important advantages in security and ITS applications. Data collection is no more limited to a fixed position along the road (like a tripod or a fixed pole), but can be performed almost everywhere within the traffic flow. Auto-Detector is a fully integrated system comprising the on-board Computer Vision and ANPR recognition subsystem, and a central supervisory station for data collection (upload-download) as well as data search and update and plate list management. The proposed mobile Auto-Detector system fits exactly the expectations and requirements of the customers.

The on-board subsystem integrates ANPR recognition functions with an effective and ergonomic man-machine interface including other service functions of communication (public or private radio link) and geo-reference (through GPS and local encoder), with optional interface to standard navigator subsystems.

On-board man-machine interface is also used to manage online communication with the control centre, to display license plates recognised during patrols and to provide alarm messages (blacklisted licence plate detected). A key issue of AutoDetector is camera sensor configuration to manage unpredictable variations in lighting conditions, from just a few lux in areas of shadow, tunnels and night operations, to more than $100 \mathrm{Klux}$ in full sunlight. The integrated acquisition system comprises a digital micro-camera coupled to a programmable high-speed pulsed LED illuminator in the near infrared spectrum to limit ambient light interference.

An important feature of Auto-Detector is its extremely easy operation that greatly improves user acceptance. When starting their mission, patrol officers are not required to perform any supplementary operations. All data-update operations are performed completely automatically over the wireless-LAN link. During patrols, officers receive and send messages from/to the Operations Centre through the available interface monitor. When a license plate in the hot-list is recognised, an alarm signal starts and the acquired digital images are immediately displayed on screen to provide the human operator evidence of the positive detection. The alarm signal is automatically sent also to the Operations Centre through the onboard communication system in real time. A marker is automatically entered on the onboard navigator map to display the position (on the navigation map) where the license plate was detected and recognised. On returning from patrols, the system automatically uploads the data it has collected to the central unit over the wireless-LAN link and then shuts the system down. The storage of vehicle transit data and images is managed according to the privacy rules established in the different countries. 

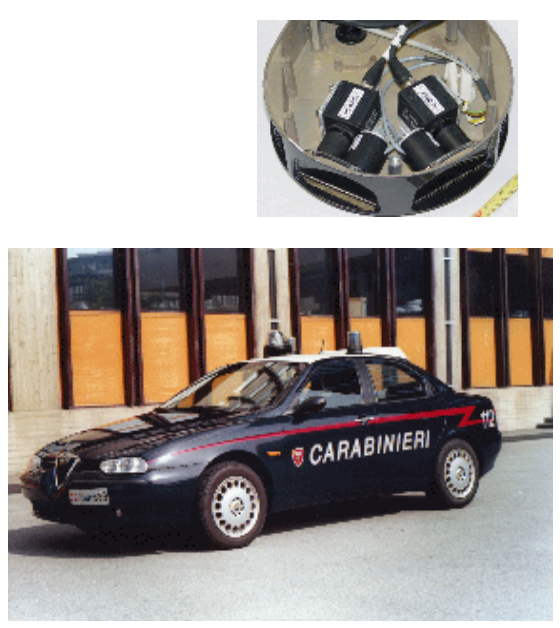
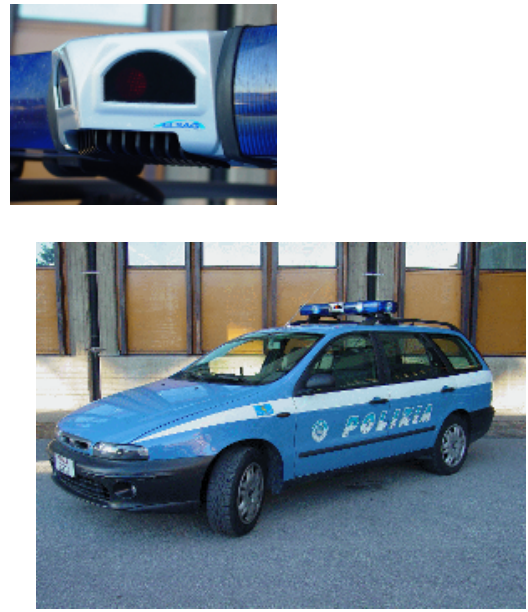

Fig. 1. Auto-Detector has been installed on-board of police patrol cars, since 2002

\subsection{Brief History of the Project}

Elsag has developed its first ANPR system at the beginning of the 90's for traffic control applications, from pay-toll highway access control (Telepass by Italian Autostrade), parking control, access control to restricted traffic areas.

The mobile Auto-Detector project started in 2001 as a technology-push by the Technology Research department on Imaging and Computer Vision with a first prototype to demonstrate feasibility of the mobile recognition function. Such early experience has pinpointed a few main problems dealing with reading performance, computational speed, sensor miniaturization, automotive requirements for the processing unit (compact and low-power consumption). At the same time the Security Institutions in Italy (Police and Carabinieri Army) were looking for advanced solutions able to read license plates on-board of patrol cars, to support the heavy (almost impossible) task of patrol crews to search hot-list vehicles in the normal traffic flow.

On March 2002 a first demonstration to the Police department has been issued. This initiative proved the great potential of the proposed system and provided some important feedback in terms of application requirements, ergonomic issues and system constraints (communication and operational procedures). The product has been protected by an international patent "Surveillance System for Homeland security through Mobile Automatic Number Plate Recognition of Vehicles in transit"; by G. Garibotto, P. Castello, E. Del Ninno, submitted to the PCT (Patent Cooperation Treaty) through all main countries (Europe, USA, Canada, South-America).

On July 2003 a demo-kit of the system was realized. It consists of two small cases. The first case contains the processing unit to be placed in the baggage compartment of the car (with power connection to the light-power 12V DC of the car). The second case contains cables and the sensor box, with magnetic support, so that it can be easily placed on top of any existing car. Man-machine interface is granted by a portable laptop PC with wireless-LAN Wi-Fi connection. In 2004 International applications 
started with a series of demonstrations that have been arranged in the international market., with participation to the most qualified international fairs. On August 2004 a joint company has been founded between Elsag and Remington Arms (US) whose name is RELES (Remington-Elsag Law Enforcement Systems) [6] to commercialize the Auto-Detector system in the US market. Since the beginning of 2005 the US market has been addressed with extremely positive results [Ohio]; moreover, additional applications have been launched in other countries (South Africa, Turkey, China, etc.). The overall direct cost of the development project has been covered by internal funding of the company but the basic competence and technology has been achieved through the participation to different national and international projects. A relevant contribution came from the Italian Ministry of Education, University and Research (MIUR) in a project led by Elsag, entitled "Neural systems for service and plant automation", corresponding to Theme 5 of the "National Program for Microelectronics and Bioelectronics" (2000-2003). Basic knowledge on Computer Vision and Image processing Technology has been developed within the European projects of ESPRIT P940 "Depth and Motion Analysis" (86-92) and more recently within the project VISOR IST -1999-10808 (2000-2002), devoted to video monitoring applications. Further investments are in progress by Elsag to support technology and product evolution, to improve recognition performance, increase processing speed, improve resolution and range of operation as well as to introduce additional services and functions to patrol car operators.

\subsection{Related Products on the Market}

At the very beginning of the project (2002) the other competitive systems were definitely less performing and quite limited (in speed and resolution). During the last year (2005) the number of competitors in the international market has been steadily increasing. Now we can identify a number of more or less competitive systems and new solutions are continuously appearing on the scene, to confirm the maturity of technology and the application.

- Auto-Find from Auto-Vu: (Canada): a system solution originally developed for parking control with visible light solutions; more recently it has been modified with infrared light source to work in all environmental conditions.

- Mobile system from Appian Technology (UK): the early solution was based on passive vision using standard video sensors inside the vehicle; more recently a new configuration based on infrared LED sensor has been introduced in the market.

口 Mobile systems from PIPS Technology (UK): a high-quality IR integrated sensory system has been developed for mobile applications to work both at high speed on the highway and at low-speed in downtown.

\section{Discussion and Conclusion}

Unfortunately, not always the most exciting research subjects bring to business success. A favourable combination of different factors is required, including a significant component of technology innovation, the identification of all relevant 
added values to the market, as well as a solution to real concrete problems. R\&D technology investment may become extremely profitable when are oriented to problem solving. Robustness and reliability and high quality of the results are also fundamental issues (product engineering and system configuration management) as well as the subjective quality (as perceived by the user). The solution must be effectively integrated within existing operational procedure and particular care must be devoted to all services of maintenance and user assistance. The technology development team must exhibit a high flexibility to adapt the proposed solution to user requirements along the development of the product, with the involvement of the user in the development phase. The company qualification and certification is extremely important. Credibility and reputation are difficult to achieve but they are very easy to lose. In this context IPR activity (patents \& publications) as well as licensing strategy must be well defined and supported.

Partnership relationships are extremely critical in the development of a new business based on R\&D innovation. It may be technical (to complement internal competence and "core" technology) as well as commercial (to support introduction especially in the international market).

The referred example of Auto-Detector is a successful combination of both technical and commercial components where the maturity of the Imaging Technology (Plate Recognition in real-time) and the nice idea to let it free to move inside the traffic flow to automatically search for the plates in the list, was properly fitting the requirements of security institutions in the new emerging field of Homeland Security.

Any new project is a different story by itself but I do hope $t$ this experience to be of some help for a better understanding of the complex issue of R\&D Technology development. Moreover it may be a stimulus for the development of new and more ambitious projects based on Computer Vision.

\section{References}

1. W.B. Rouse, "R\&D/Technology Management: a framework for Putting Technology to Work", IEEE Trans. On Systems, Man and Cybernetics, part C: applications and Reviews, vol 28, n.4 Nov. 1998, pp.501-515.

2. T. J. Allen "Managing the flow of Technology", Cambridge, MA; MIT Press, 1977.

3. The European Information Society Technologies Prize, EEC IST-prize, www.ist-prize.org.

4. T.K. Sung, B.S Kang, S K Lee, "Study on Characteristics of Technology Transfer in Venture Business", Proceedings of the $34^{\text {th }}$ Hawai Int. Conf. On System Sciences, 2001.

5. G.Garibotto, "Auto-Detector: Mobile Automatic Number Plate Recognition", Handbook of Pattern Recognition and Computer Vision, ed. C.H.Chen \& P.S.P. Wang, chap 5.6, pp. 601-618

6. RELES, Remington-Elsag Law Enforcement System, http://www.remington-elsag.com/ Home.htm, 